\title{
Abnormal and Behavioural Psychology
}

\section{Why Young Patients with Cancer Require Different Coping Tools?}

\author{
Calderon $\mathrm{C}^{1^{*}}$, Jimenez-Fonseca $\mathrm{P}^{2}$, Carmona-Bayonas $\mathrm{A}^{3}$ and Jara $\mathrm{C}^{4}$ \\ ${ }^{1}$ Department of Clinical Psychology and Psychobiology, Faculty of Psychology, University of Barcelona, Spain \\ ${ }^{2}$ Medical Oncology Department, Hospital Universitario Central de Asturias, Oviedo, Spain \\ ${ }^{3}$ Medical Oncology Department, Hospital Universitario Morales Meseguer, Murcia, Spain \\ ${ }^{4}$ Medical Oncology Department, Hospital Universitario Fundación Alcorcón, Madrid, Spain
}

"Corresponding author: Calderon C, Professor of Psychology, Department of Clinical Psychology and Psychobiology, Faculty of Psychology, University of Barcelona, Spain, Tel: +34933125107; Fax+34934021362; E-mail: ccalderon@ub.edu

Received date: February 22, 2017, Accepted date: February 28, 2017, Published date: March 07, 2017

Copyright: ( 2017 Calderon C, et al. This is an open-access article distributed under the terms of the Creative Commons Attribution License, which permits unrestricted use, distribution, and reproduction in any medium, provided the original author and source are credited

\section{Manuscript Organization}

The medical field has made great advancements in recent decades, but even one in every two men and one in three women will suffer from cancer throughout their lives [1]. Furthermore, cancer was the leading cause of death due to illness in the adolescent and young adult (18-39 year old) population [2]. Despite these figures, no one thinks he is close to suffering from cancer. We prefer to think that it is a disease related to some other collective that we perceive as distant. We are not prepared to deal with the processes involved in the diagnosis and treatment of cancer. Therefore, it is a disease that poses challenges beyond the medicine itself and that forces to take action in relation to the personal and social environment of the patient, measures that will probably transform their present and future lifestyle. This becomes especially relevant if we focus on cancer in young patients [3].

The cancer does not warn, appears suddenly and in the least thought: a periodic review, a slight symptom that does not remit. Although, it is an unwanted guest and for whom we have not booked any appointments, we will have to place it for a long time in the center of our life and this is especially complicated in young people.

There is no optimal age for cancer, but during youth it may be even more difficult to deal with this disease, since by nature young people challenge established rules, wish to experiment, live by their own standards and the cancer sends them an opposite message. Cancer forces young patients to reconsider their lives in many ways, their projects, plans and independence may be postponed for an indefinite period.

The loss of independence and the need to adjust to deal with cancer or with lasting effects from cancer cure require to modify many life plans, which will generate logical feelings of frustration, leading patients to seek some isolation, generating sadness, isolation, hopelessness and even anger. This resentment towards the world and its designs often gives way, sooner or later, to a confrontation with resignation, accepting reality and shifting shyly towards some optimism.

Medical tests to detect and treatments to combat leukemia, lymphoma, testicular cancer (the most common between 15-24 years old), breast cancer or melanoma (the most common between 25-39 years old), show an unknown world full of uncertainty and medical decision making with a great vital impact. Fear, alienation and solemnity accompany many stages of the young patient [4].

The paradox of having better health and at the same time being in a situation of great risk makes the young patient stagger and shake of spirit throughout the treatment, going from the hope felt by the improvements, to the discouragement whenever there is a stumbling block. From this confrontation with one's own death, there is an unexpected but common personal growth for young patients who have overcome cancer: their lives have changed and taken on a dimension that they did not have before. His way of seeing the future will have a more transcendental nuance, facing every moment as the last, living the present with greater awareness and fullness.

This spiritual or transcendent dimension, as well as some changes in lifestyle (food, healthy habits, sports, etc.) will be essential components to strengthen the patient's resilience, that emotional capacity to live adverse situations and overcome them with hope and optimism.

Social, affective, family and labour relationships will be affected in some way: the experience of an extreme situation will represent a symbol in the life of a young patient, testing family, partners, friends and above all him selves. Perhaps the treatment has changed their physical appearance, their mood, sexuality, reproductive capacity, appetite or physical strength. They will have to learn to live with it and in this way; health professionals and their nearest trustworthy people will be decisive.

The physician's communication with the patient, the agility and efficiency in the procedures and the shared decision-making become fundamental elements that will affect the satisfaction and coping of the patient [5]. Sharing all these emotions is a privilege and a responsibility for those who are close to these patients and accompany them in their therapeutic process. Together with the treatment of cancer we must rely on psychology as knowledge that will facilitate their labor, social and affective insertion, will guide family, caregivers and friends in the way of organizing this new life.

There have been many advances that reduce mortality [6], and the negative consequences of the disease. Nevertheless, it is essential to deepen and extend the psychological and social assistance to these patients, even once discharged.

Some of them will suffer from financial difficulties, higher medical and pharmaceutical costs [3], limitations on insurance or medical care, changes in their physical appearance and body image, work restrictions, wages or difficulties in returning to their former jobs. The personal growth they have experienced will make the vast majority of them better people for life. In this, the professionals who care for them can influence in a decisive way and for this reason it is important to know better the emotional state, the quality of life and the style of coping with the diagnosis and throughout the process[7].

In order to determine the extent of psychological distress in young patients with cancer, a multidisciplinary team of health professionals 
Citation: Calderon C, Jimenez-Fonseca P, Carmona-Bayonas A, Jara C (2017) Why Young Patients with Cancer Require Different Coping Tools?. Abnorm Behav Psycho 3: 129. doi:10.4172/2472-0496.1000129

Page 2 of 2

including myself, from 13 Spanish hospitals have designed and participated in the NEOCOPING project, funded by SEOM in 2015-16, whose Preliminary studies results have shown that young patients presented significantly a greater psychological distress and anxiety than older ones, as well as differing in the type of coping strategy applied, focused on the fighting spirit in young people and resignation in the elderly. Interdisciplinary research in fields such as this one, becomes more necessary, effective and strategic in a globalized world, which seeks to minimize the presence of cancer and its impact on future generations.

\section{References}

1. Kinahan KE, Sanford S, Sadak KT (2015) Models of Cancer Survivorship Care for Adolescents and Young Adults. Semin Oncol Nurs 31: 251-259.

2. Nass SJ, Beaupin LK, Demark-Wahnefried W (2015) Identifying and Addressing the Needs of Adolescents and Young Adults With Cancer: Summary of an Institute of Medicine Workshop. Oncologist 20: 186-195.
3. Ambrose K, Arvey SR, Casillas JN (2016) Financial and work related impact of cancer in young adult (YA) survivors. J Clin Oncol 34: 15.

4. Agostino NM, Pond GR, Aubin S (2016) Neurocognitive function and psychological distress in young adults (YA) with cancer. J Clin Oncol 34: 199.

5. Kaul S, Fluchel M, Parmeter CF, Spraker HL (2016) Healthcare utilization and quality among survivors of adolescent and young adult cancer. J Clin Oncol 34: 21.

6. Kehl KL, Landrum MB, Arora NK (2015) Shared decision making in cancer care: The association of actual and preferred decision roles with patient-reported quality. JAMA Oncol 1: 50-58.

7. Zeuner R, Frosch DL, Kuzemchak MD, Politi MC (2015) Physicians' perceptions of shared decision-making behaviours: a qualitative study demonstrating the continued chasm between aspirations and clinical practice. Health Expect 18: 2465-2476. 